Association for Information Systems AIS Electronic Library (AISeL)

Wirtschaftsinformatik Proceedings 2001

Wirtschaftsinformatik

September 2001

\title{
Intelligent Brokering of Environmental Information with the BUSTER System
}

Heiner Stuckenschmidt

Intelligent Systems Group, Center for Computing Technologies, University of Bremen, heiner@tzi.de

Thomas Vögele

Intelligent Systems Group, Center for Computing Technologies, University of Bremen, vogele@tzi.de

Ubbo Visser

Intelligent Systems Group, Center for Computing Technologies, University of Bremen, visser@tzi.de

Ryco Meyer

Intelligent Systems Group, Center for Computing Technologies, University of Bremen, ryco@tzi.de

Follow this and additional works at: http://aisel.aisnet.org/wi2001

\section{Recommended Citation}

Stuckenschmidt, Heiner; Vögele, Thomas; Visser, Ubbo; and Meyer, Ryco, "Intelligent Brokering of Environmental Information with the BUSTER System" (2001). Wirtschaftsinformatik Proceedings 2001. 54.

http://aisel.aisnet.org/wi2001/54

This material is brought to you by the Wirtschaftsinformatik at AIS Electronic Library (AISeL). It has been accepted for inclusion in Wirtschaftsinformatik Proceedings 2001 by an authorized administrator of AIS Electronic Library (AISeL). For more information, please contact elibrary@aisnet.org. 
In: Buhl, Hans Ulrich, u.a. (Hg.) 2001. Information Age Economy; 5. Internationale Tagung Wirtschaftsinformatik 2001. Heidelberg: Physica-Verlag

ISBN: 3-7908-1427-X

(C) Physica-Verlag Heidelberg 2001 


\title{
Intelligent Brokering of Environmental Information with the BUSTER System
}

\author{
H. Stuckenschmidt, T. Vögele, U. Visser and R. Meyer \\ Intelligent Systems Group, Center for Computing Technologies, University of \\ Bremen
}

\section{Motivation}

Many application areas of information systems share the need to store and process large amounts of diverse data, which is often geographically distributed. This implies that in order to make new data available to the system these data has to be transferred into the system's specific data format. This is a very time consuming and tedious process. Data acquisition, automatically or semi-automatically, often requires large-scale investment in technical infrastructure and/or manpower. These obstacles are some of the reasons behind the concept of information sharing. Information sharing is attractive because in order to supplement an existing data basis remote information can be accessed by systems. The advantages of successful information sharing is thus obvious for many reasons:

- Quality improvement of data due to the availability of large and complete data.

- Cost reduction resulting from multiple use of the existing information sources.

- Avoidance of redundant data and conflicts that can arise from redundancy.

However, in order to establish efficient information sharing, difficulties arising from organizational and competence questions, as well as many other technical problems have to be solved. First, a suitable information source must be located which contains the data needed for a given task. Once the information source has been found, access to the data therein has to be provided. Furthermore, access has to be provided on a technical and informational level. In short, information sharing not only needs to provide full accessibility to the data, it also requires that the accessed data may be interpreted by the remote system. While the problem of providing access to information has been largely solved by the invention of largescale computer networks, the problem of processing and interpreting retrieved information remains an important research topic (Visser, Stuckenschmidt et al. 2000). 


\section{The BUSTER Approach}

In systems with a large number of available data sources, it is seldom trivial to find the right set of data for a given task. If, for example, an information request is submitted to an information broker, the broker has to decide which of the registered sources it should use to answer the request. The BUSTER approach (Bremen University Semantic Translator) addresses these questions by providing a common interface to heterogeneous information sources in terms of an intelligent information broker (http://www.semantic-translation.de). The user can submit a query request to a network of integrated data sources. In this query phase several components interact at different conceptual levels.

Metadata, i.e. data describing a data source, are often used to organize and manage large collections of data sources. Typically, such metadata catalogues are based on standardized meta data formats like the Dublin Core (http://dublincore.org). In the BUSTER approach, each data source is represented by a specific ontology, the socalled source ontology (Stuckenschmidt, Wache et al. 2000). It contains an explicit description of the concepts covered by the data source together with information about the structural and syntactic details of the data source. User queries are matched against different source ontologies. If the matching succeeds, the broker establishes a connection to the actual information source. If the matching fails, the broker decides that there is no valuable information available and tries different information sources (Vögele, Stuckenschmidt et al. 2000)

On the syntactic level, so-called wrappers are used to establish a communication channel to the data source(s) found, which is independent of specific file formats and system implementations. Wrappers exist for specific file- or data-format. For example, generic wrappers may exist for ODBC data sources, XML data files, or specific GIS formats. Still, these generic wrappers have to be configured for the specific requirements of a data source.

The mediator on the structural level uses information obtained from the wrappers and combines, integrates, and abstracts them. BUSTER allows the use of different mediators which are configured by transformation rules. These rules describe in a declarative style, how the data from several sources can be integrated and transformed to the data structure of the original source.

At the semantic level, we use two specialized tools for solving the semantic heterogeneity problems based on the ontologies which describe the contents of the information sources. Both tools are responsible for the translation from one vocabulary to the other (context transformation), i.e. transforming data from the context of one information source to the context of another information source. There are several ways how the context transformation can be applied. In BUSTER we consider the functional context transformation and context transformation by re-classification. 


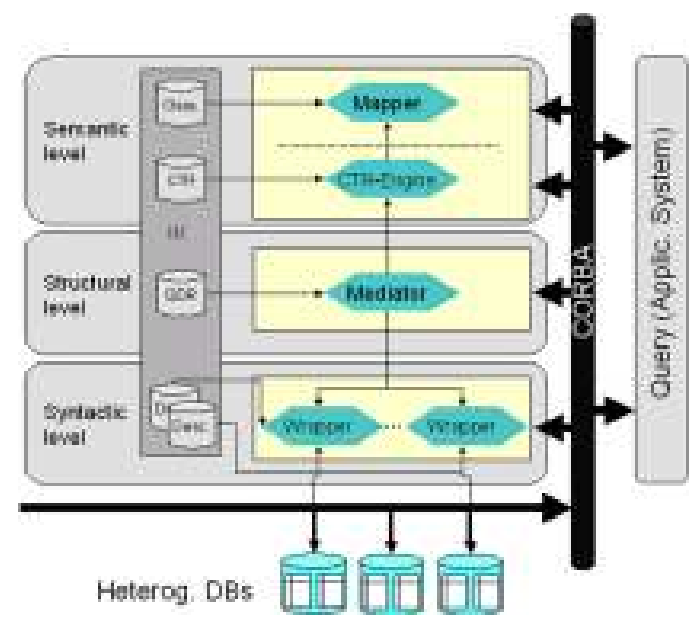

Figure 1: The BUSTER Approach

BUSTER uses the OIL language for the description of contents-related metadata. The language has been developed in the context of the On-To-Knowledge Project (www.ontoknowledge.org) as a proposal for a language for the specification and exchange of ontologies (Fensel et al. 2000). OIL tries to provide a core set of features that have been widely accepted to be useful for the description of vocabularies and terminologies. OIL combines object-oriented modelling primitives, reasoning facilities from Description Logics and a tight interaction with meta-data standards on the web such as RDF and XML. We used OIL to build a semantic context model of our example data by identifying a set of common properties which can be used to define a land use class.

\section{Retrieving Spatially Related Information}

In the field of environmental science, most documents and other data sources have some sort of spatial connotation. Obviously all geo-spatial data, i.e. data which are typically handled by GISs (Geographic Information Systems), refer to a specific geographic area. But also for non-spatial data sources, such as reports, documents and databases, references to geographic locations are typically important attributes. For example, a report about the installation of new groundwater monitoring wells very likely refers to a specific (geographic) investigation area. Consequently, spatial attributes are important for both information retrieval and the description and management of data sources with the help of metadata catalogues. However, most online systems, like metadata catalogues and other browser-based information retrieval systems, offer only very little to represent and query the complex relations of data sources and their respective locations in space. 
To overcome some of the shortcomings of existing approaches described in (Vögele and Stuckenschmidt 2001), we use qualitative spatial relations for information retrieval. We use the spatial configuration depicted in Figure 2 to illustrate the determination of spatial relevance on the basis of topological relations. In our example, we are concerned with different project areas in a city. The project areas are spatially associated with specific districts using topological relations.

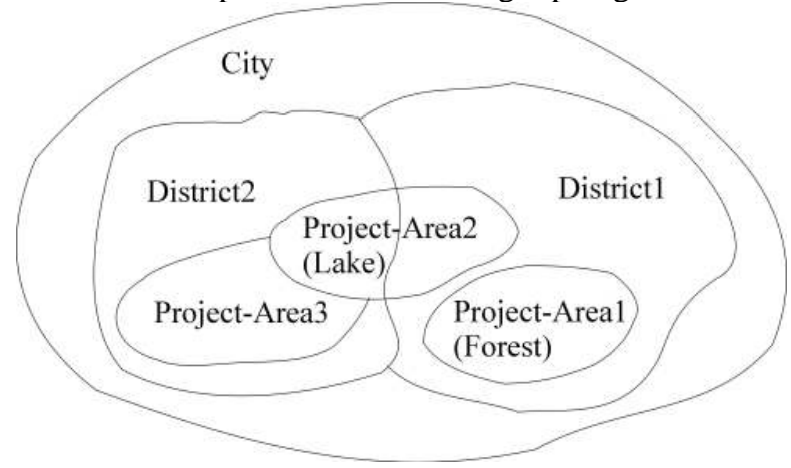

Figure 2: Spatial Configuration of the Example

The first relation we use to refer to project areas is spatial containment. Project Area 1, for example, is contained in District 1, while project Area 3 is contained in District 2 . We further declare, that every area which his contained in another area is automatically spatially relevant with respect to the including area. This can be achieved by defining a relation contained-in as a special case of a relation definitely-spatially-relevant. Using the OIL language, we can define contained-in as well as its mathematical properties (i.e. transitivity) in the following way:

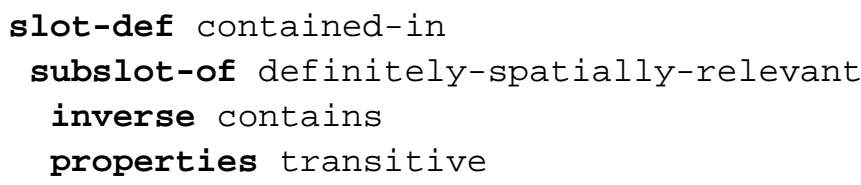

We can now use the relevance relation to retrieve areas which are spatially relevant to District 1. Using the FaCT reasoner interface, we can formulate a query Q1 for areas spatially relevant to District 1 in the following way:

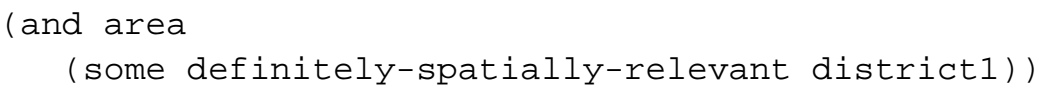

Not surprisingly, the result of this query is Project Area 1, because it is contained in District 1. However, Project Area 2 may also be of interest when querying areas related to District 1, because it is at least partially contained in District 1. We cover this kind of relevance by using another topological relation, namely partial overlap. As we are not absolutely sure that Project Area 2 is really relevant, we use a relation probably-spatially-relevant to describe a weaker level 
of relevance. Again, we define relevance in terms of topological relations by stating that partial overlap is a special kind of spatial relevance. The OIL definition of the relation partially-overlapping is the following:

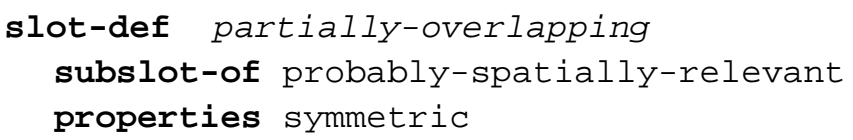

We further define that, because of its weaker character, our previous notion of relevance also falls under this new relation. The result of a query Q2 searching for areas probably-spatially-relevant to District 1 consists of Project Area 1 and Project Area 2, because the latter overlaps with District 1.

As mentioned above, areas in the neighborhood may also be of interest. We therefore include a further level of spatial relevance based on neighborhood defined by the relation connected-to. We assume that this third level of spatial relevance is even weaker than the ones introduced above, because our notion of connectedness implies that there is no overlap.

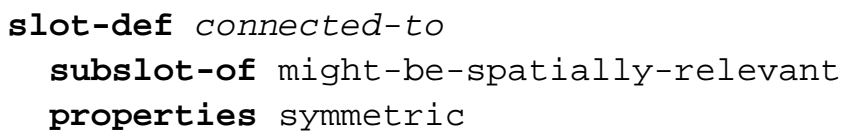

Using this notion of spatial relevance, we still find Project Areas 1 and 2. Additionally, we get District 2 as an area spatially relevant to District 1 . However, using OIL it is not possible to derive the spatial relevance of Project Area 2, which is contained in the relevant area District 2, in a straightforward way because we cannot chain relations in order to determine spatial relevance.

\section{Semantic Translation of Retrieved Objects}

We carried out a case study on semantic information integration based on a reallife problem from the field of geographic information processing. Geographical information systems normally distinguish different types of spatial objects. Different standards exist for specifying these object types. Since there is more than one standard, various catalogues compete with each other. To date, no satisfactory solution has been found to integrate these catalogues. In our evaluation we concentrate on different types of land-use that might be used as a criteria for information retrieval.

\subsection{Information Sources}

The ATKIS catalogue (AdV 1998) is an official information system in Germany. It offers digital landscape models with different scales from 1:25.000 up to 
1:1.000.000 with a detailed documentation in corresponding object catalogues. We use the large-scale catalogue OK-1000. This catalogue offers several types of objects including definitions of different types of areas. Figure 3 shows the different types of areas defined in the catalogue.

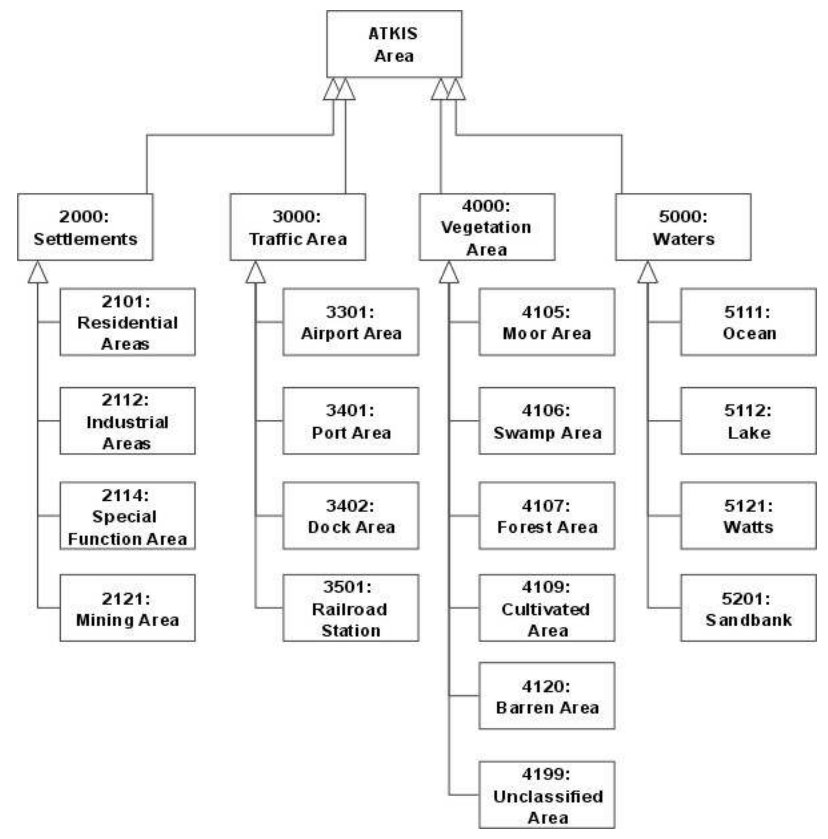

Figure 3: Class Hierarchy of the ATKIS-OK-1000 Classification

CORINE land cover (EEA 1997-1999) is a deliverable of the CORINE program the Euro-pean Commission carried out from 1985 to 1990. It defines nomenclatures and methodologies which are now used as the reference in the areas concerned at the community level. The nomenclature developed in the CORINE programme can be seen as another catalogue because it also defines the taxonomy of area types (see Figure 4) with a description of characteristic properties of the different land types. 


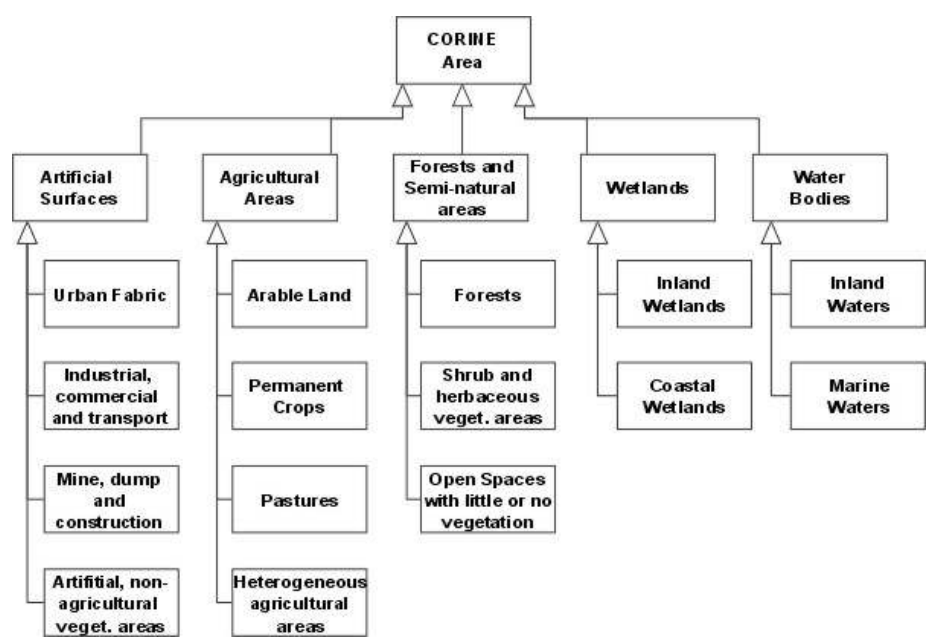

Figure 4: Class Hierachy of the CORINE Landcover Nomenclature

The taxonomies of land-use types in fgures 3 and 4 illustrate the context problem mentioned in the introduction. The set of land types chosen for these catalogues are biased by their intended use: while the ATKIS catalogue is used to administrate human activities and their impact on land use in terms of buildings and other installations, the focus of the CORINE catalogues is to describe the state of the environment in terms of vegetation forms. Consequently, the ATKIS catalogue contains fine-grained distinctions between different types of areas used for human activities (i.e. different types of areas used for traffic and transportation) while natural areas are only distinguished very coarsely. The CORINE taxonomy on the other hand contains many different kinds of natural areas (i.e. different types of cultivated areas) that are not further distinguished in the ATKIS catalogue. On the other hand, areas used for commerce and traffic are summarized in one type.

Despite of these differences in the conception of the catalogues, the definition of the land-use types can be reduced to some fundamental properties. We identified six properties that can be used to define the classes in both catalogues. Beside size and general type of use (e.g. production, transportation or cultivation), the kinds of structures built on top of an area, the shape of the ground and natural vegetation as well as kinds of cultivated plants and three topological relations between area types are discriminating characteristics.

\subsection{Translation Experiments}

Using the definitions mentioned above, we performed a series of translation experiments. Some of these results are described below. The basis for our experiment was a small CORINE landcover data set containing information about the 
town 'Bad Nenndorf' in Lower-Saxony. This data set is available from the German environmental agency in different formats and classifications and can therefore be used to compare and evaluate results. The data set contains areas of five different types, namely

- Discontinuous urban fabric

- Non-irrigated arable land

- Pastures

- Broad-leaved forest

- Mineral extraction site

Except for 'pastures' all these types do not directly correspond to concepts defined in our model. They are rather sub-types or special cases of the concepts we defined. Consequently, we can use the definitions from the CORINE ontology, but we have to refine the descriptions according to the additional information that is available in terms of a further specialization of the concepts.

One of the data-sets used in the case study is classified as 'broad-leaved-forest' which is a sub-class of the CORINE concept 'forest' mainly consisting of broadleaved trees. We get a description of this concept by adopting the definitions of the super-classes 'forests' and 'forests-and-semi-natural-areas' and specializing the 'has-value' constraint on the 'vegetation' slot from 'trees' to 'broad-leaved-trees'.



In this case of 'broad-leaved-forest' we got the correct result for the target hierarchy already with the first ad hoc definition of the concept to be classified. The subsumers from the target hierarchy are:

- VEGETATION-AREA

- FOREST-AREA (direct subsumer)

Looking at the target hierarchy, we can see that this is exactly the position we expected. So, we can say that at least for this case the semantic translation problem could be solved in a straightforward way using OIL and the FaCT reasoner. 


\section{$5 \quad$ Integrating Spatial and Terminological Matching}

Type information about information items to be retrieved can be organized using structured concept hierarchies like thesauri and ontologies. Above we argued that description logics are very well suited for the formalization of such concept hierarchies as well as for concepts of spatial relevance. Therefore, using description logics to encode both spatial relations and type information allows for the specification and fine-tuning of integrated queries.

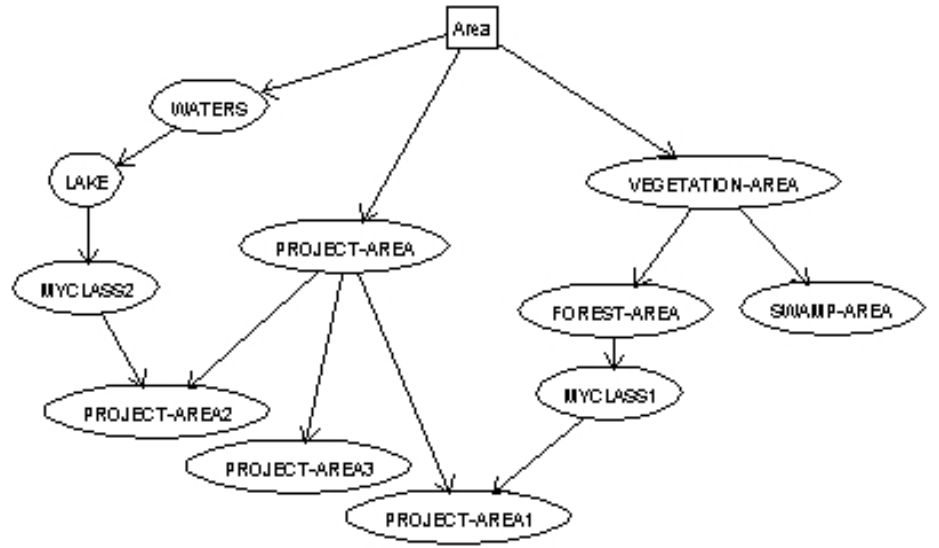

Figure 5: Sub-class Relations Computed by the FaCT Reasoner

In order to include terminological information in queries, we further describe the project areas using class definitions and defining the areas to be instances of these classes. We might for example know that Project Area 1 has solid ground and its vegetation consists of oaks. Using OIL we can capture this knowledge in the following class definition.

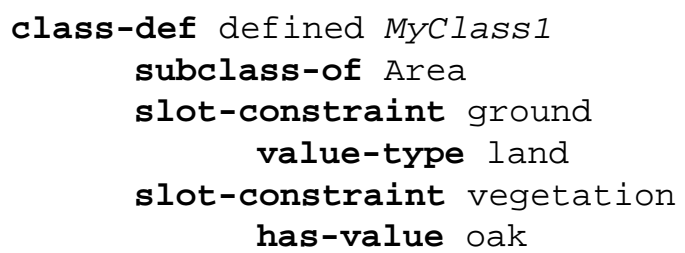

Using the FaCT reasoner, we can automatically determine the super-class of this definition and therefore the terminological category that Project Area 1 belongs to. In our case we derive that Project Area 1 is a "forest", because its class definition constitutes a special case of the following general definition of a "forest area":

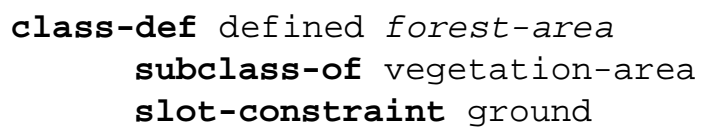




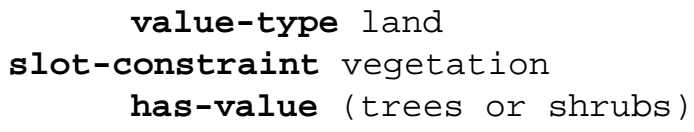

In the same way, we model the class of Project Area 2 in such a way that it can be derived to belong to the category "lake" (see Figure 5 for a complete class hierarchy of the example). We can use this terminological information to find answers to more sophisticated queries. The first possibility is to restrict the type of areas we are interested in. For example, we can ask for "forest areas" that might be spatially relevant to District 1 :

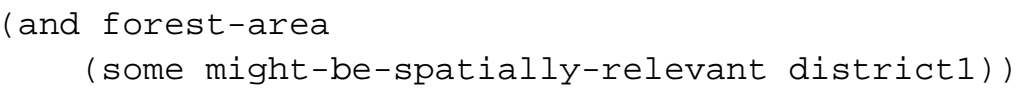

Using this additional type restricting, the result of the query is reduced to Project Area 1 , because the other areas also relevant to District 1 are not of type forest area.

Another application of terminological information is not to seek for areas that are relevant to a specific area, but rather to a specific class of areas. For example, we can ask for areas that are spatially relevant to "lakes" in general. The corresponding query is the following:

(and area

(some might-be-spatially-relevant Lake))

Because the logic reasoner is able to infer that Project Area 2 is a "lake", we retrieve all areas that are spatially related to Project Area 2. In our case these are Districts 1 and 2 because they overlap with Project Area 2 and, because of its connectedness to Project Area 2, also Project Area 3.

\section{The BUSTER System}

A first prototype of the BUSTER approach has been implemented. The current functionality includes ontology-driven search for information sources as well as schematic integration of geographical information sources. The prototype is built upon tools which were developed at the university of Manchester to facilitate the use of the OIL language:

- FaCT, a logical reasoning service that can be used to check ontologies for consistency and for computing subclass relations not explicitly contained in the ontology (Horrocks 1999).

- The Ontology Editor OILed providing a graphical interface for the definition of complex ontologies and a direct interaction with the FaCT reasoner in a client-server architecture 
The editor is used to create meta-data models as well as context definitions used in the semantic translation step.

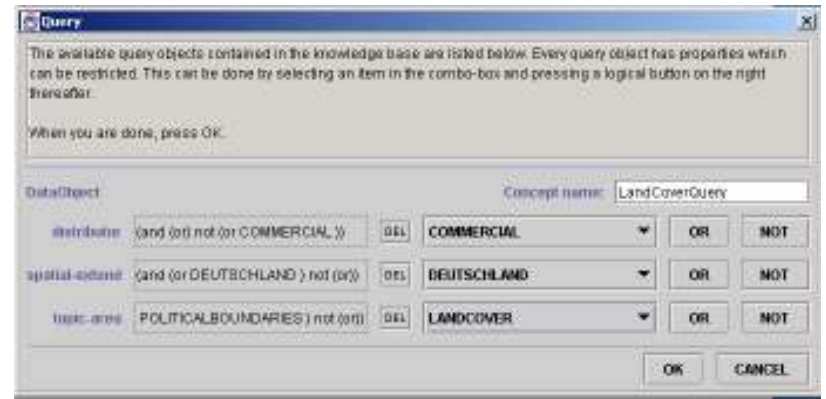

Figure 6: The BUSTER Query Interface

Figure 6 shows the query interface of the prototype. The interface is dynamically created on the basis of a query model. The user is asked to restrict the defining properties of the class in order to restrict the set of all information sources to those of interest. Currently, the FaCT reasoner is the main inference engine of the BUSTER system. The resulting class definition is passed to the reasoner that places the query in a hierarchy of classes. Each class is a surrogate for an information source. All classes placed in the subtree rooted at the query class are returned, because they fulfil the constraints defined in the query. The result of the query phase is a list of all information sources matching the query. Figure 7 shows the result of a query targeting at land-use data about a special region in Lower Saxony.

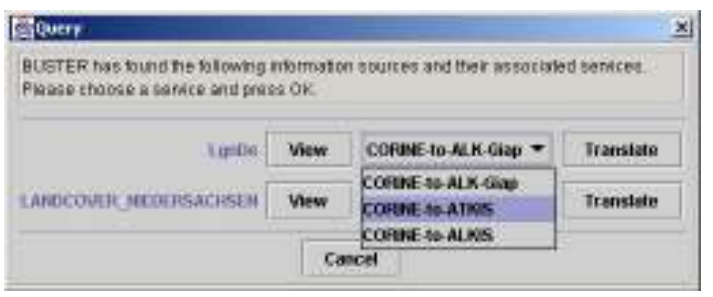

Figure 7: Results of the Query in the BUSTER Transformation Screen

The user can now either directly view the information as a GIF image or define a target file format the information source should be converted to. Currently, in both cases the Feature Manipulation Engine FME, a conversion tool for geographical data formats is used to create the output format. 


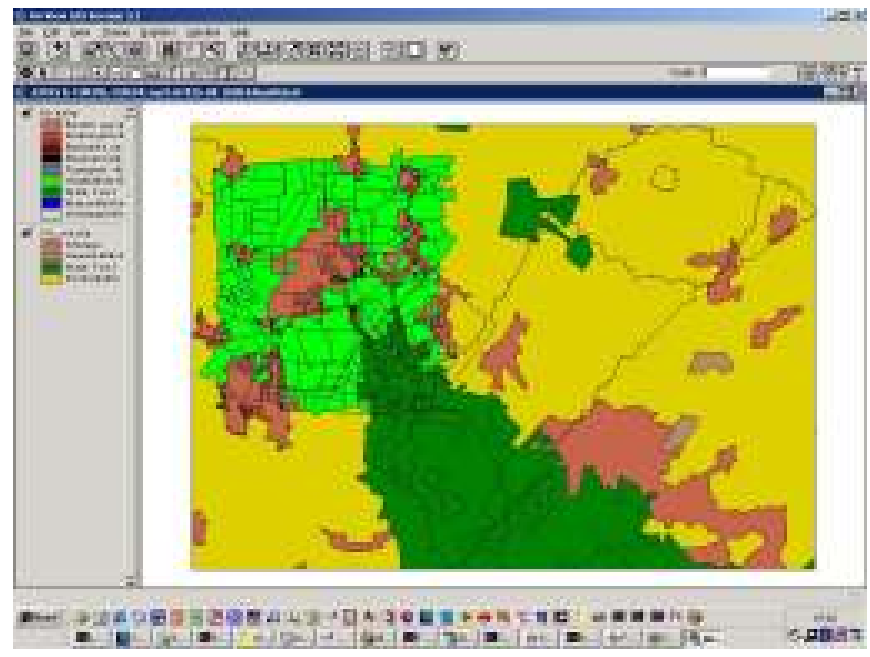

Figure 8: Integrated View on Land-Use Information

In the near future the system will be connected with the MECOTA mediator (Wache 1999), a general translation system which can be used to convert arbitrary data structures and is also capable of performing translations on the semantic level. We also aim at the integration of additional reasoning methods in addition to the $\mathrm{FaCT}$ reasoner in order to allow more flexible search and integration.

\section{Discussion}

We presented the BUSTER approach for the intelligent brokering of complex, spatially related information, explained the knowledge-based technology underlying the approach, and briefly described a prototypical implementation. The ability of the BUSTER system to combine terminological with spatial reasoning makes it an ideal platform for the exchange of environmental information which is normally related to a geographic location, and frequently uses scientific vocabularies from different disciplines that need to be integrated when searching for a special piece of information. Currently, the system is still in the development phase, but first experiences have been made that show that in principle the approach can be successfully applied. 


\section{References}

ADV, 1998, Amtliches Topographisch Kartographisches Informationssystem Atkis. Tchnical report, Landesvermessungs-amt NRW, Bonn.

EEA, 1997-1999, Corine land cover. Technical Guide. Technical report, European Environmental Agency. ETC/LC, Euro-peanTopic Centre on Land Cover.

Fensel, D., I. Horrocks, et al. (2000). OIL in a Nutshell. 12th International Conference on Knowledge Engineering and Knowledge Management EKAW 2000, Juan-les-Pins, France.

Horrocks, I. (1999). FaCT and iFaCT. Proceedings of the International Workshop on Description Logics (DL'99). P. Lambrix, A. Borgida, M. Lenzerini, R. Möller and P. Patel-Schneider: 133-135.

Stuckenschmidt, H., H. Wache, et al. (2000). Enabling Technologies for Interoperability. Workshop on the 14th International Symposium of Computer Science for Environmental Protection, Bonn, Germany, TZI, University of Bremen.

Visser, U., H. Stuckenschmidt, et al. (2000). Using Environmental Information Efficiently: Sharing Data and Knowledge from Heterogeneous Sources. Environmental Information Systems in Industry and Public Administration. C. Rautenstrauch. Hershey, PA, IDEA Group.

Vögele, T., Stuckenschmidt, H, et al. (2000). Towards Intelligent Brokering of Geoinformation. Urban and Rural Data Management (UDMS), Delft, Electronic.

Vögele, T, Stuckenschmidt, H (2001) Enhancing Gezeteers with Qualitative Spatial Concepts. In Proceedings of the Workshop on Hypermedia in Environmental Protection, Ulm.

Wache, H. (1999). Towards Rule-Based Context Transformation in Mediators. International Workshop on Engineering Federated Information Systems (EFIS 99), Kühlungsborn, Germany, Infix-Verlag. 